\title{
Study on Multi-Depot Collaborative Transportation Problem of Milk-Run Pattern
}

\author{
Zhenkai Lou, Zhenzhen Li, Lieying Luo and Xiaozhen Dai
}

Wenzhou Business College, Wenzhou, China

\begin{abstract}
Analyze the relevance between Milk Run mode and collaborative transportation problem, put forward collaborative transportation problem of multiple-depot on Milk Run mode under the supply and demand separate nodes, consider the value of transport and transport costs, introduce the concept of node - arc flow, by comparing the size of traffic flow determine nodes collection, and then constructed multi-transport model of the problem. Considering one-way pickup and delivery closed, construct two-stage algorithm model, use dynamic programming recursive solution to determine the best route to pick up, and then solving delivery routing problem with different start and return point based on geometric method of Cosine. Finally use a numerical example illustrates the effectiveness of the algorithm and reasonable model.
\end{abstract}

\section{Introduction}

Transportation is the essential means to ensure the orderly conduct of production and life. There is a proverb "to get rich first build roads", which is fully demonstrates the promotion of transport on the economy. Transportation costs accounting for up to half in total logistics costs. A large part of the reason is the lack of cooperation among enterprises, resulting in insufficient capacity or load phenomenon occurs frequently. In this context, it is very important strengthen the cooperation between enterprises, through collaborative transportation combined transport tasks, integrate transport resources, thus reducing transport costs. In collaborative transportation, cost allocation, delivery order, route planning, etc are the focus of the enterprise. Collaborative transportation is the development and the important content of collaborative logistics operation mode in recent years. Collaborative transportation demands relatively independent enterprise work together, share logistics resources and logistics information and customer requirements to reduce the traffic light, and reduce overall logistics costs, which improving performance and the service level of transportation purposes. This paper research the suppliers and demanders distribution in different regions with collaborative transportation problem of less-than-one carload logistics.

Literature [1] summarize the situation of vehicle mixed load, limit load and full coverage etc. about collaborative transportation problem, by constructing a two-stage greedy algorithm to solve the problem of largescale multi-coordinated transport depot fully loaded. Algorithm consider the radius of customer distribution and route design, and it can fit actual situations well. But it only consider the total transport mileage without considering the level of logistics services, the value of goods, etc., so the research is one-sided.

Literature [2] analyze the problem of collaborative transportation route optimization in straight-through transportation, a single point and two points of transit models, built integer programming model of transportation costs and achieved good results with using Lagrangian relaxation algorithm. Literature [4], [5] research collaborative transportation cost control from the perspective of select transit point and route coverage. Literature [7] take the efficiency of the distribution into key account, by choosing a reasonable set of nodes to establish with Hybrid Synergy distribution model to improve the client base's distribution levels. The disadvantage is not considered cost factor, and also lack scientific evidence of the number of set point. A single distribution point is often unable to meet the set requirements in reality of large-scale cooperative distribution problems.

\section{Question and models}

Coordinated transport refers to multiple transportation enterprises that according to certain organization integrated transportation tasks and resources, reasonable schedule the transportation resources, collaborative optimization is carried out on the transport task allocation so as to reduce the total cost and increase the enterprise profit and competitiveness. As for collaborative transportation now are very seriously, and has made remarkable benefit in practice.

First, compare traditional transportation with collaborative transportation. Traditional transportation 
refers to each enterprise only consider their own transport tasks, namely do the job in the form of single node. The collaborative transportation refers to a number of enterprises need to complete a number of tasks transportation, vehicles set out from a node, shipping and delivery tasks for a plurality of transport tasks and finally return to the yard.

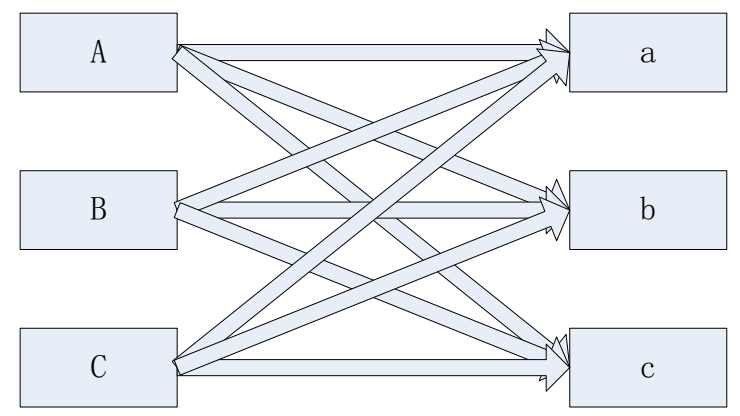

Figure 1. Traditional transportation model.

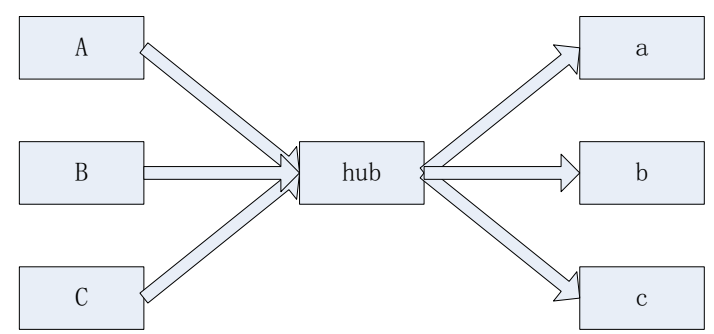

Figure 2. Collaborative transportation in distribution center.

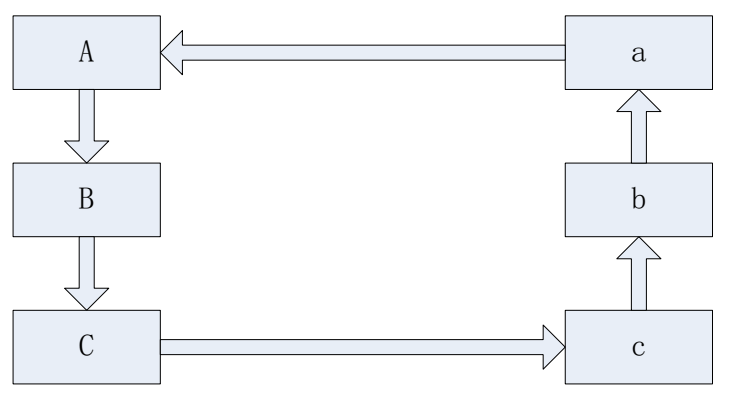

Figure 3. Milk run mode.

This paper adopts the model of the cooperative transport in Fig. 3. In collaborative transportation of different the shipper, the carrier will transport business integration in the process of optimization, and the participant enterprises must carry out individual decisions, collaborative alliance also must carry out the overall decision. Individual decision making and the overall decision-making process of the alliance, need to arrange a schedule based on the path of transport vehicles. At the same time, the cooperative transport in a number of important issues, such as participating in the mission of the organization combinatorial auction bid, collaborative design problems of transportation costs and benefits of allocation strategies, must be oriented to collaborative transportation vehicle routing problem based on optimization results.

To build the model convenient, make the following assumptions with the problems:

(1) The geographical location of each node is known, node's mileage expressed by linear distance;

(2) Each node is taken delivery and only once;
(3) All the tasks have been confirmed before shipping, the supply and demand situation is no longer change;

(4) The source of supply and demand are scattered in different areas;

(5) As our emphasis is sporadic freight transportation, every car's transport tasks are less than capacity;

(6) As a result of the gas is convenient, there are no biggest form distance constraints of the vehicle.

In the collaborative transportation process which by one or more business enterprises to take a common transport task, the smooth conduct of the premise is to adopt a uniform standard transport costs. The shipping costs only depends on the volume, weight and pick up of yard goods, and goods independent of the actual value, defined as the value of transport. The transportation costs only associated with transport mileage. Optimization goal of this paper is to make the maximum revenue of synergies transport, namely the difference costs between transport and transport of the value. Model as follows:

$$
\begin{gathered}
\max Z=\sum_{i \in M} p_{i}-q \sum_{l \in \mathrm{L}} \sum_{\mathrm{k} \in K} \sum_{\mathrm{i} \in \mathrm{O}} \sum_{j \in \mathrm{O}} c_{i j} x_{i j}{ }^{l k} \\
\text { s.t. } \sum_{i \in \mathrm{L}} \sum_{j \in \mathrm{M}} x_{i j}{ }^{l k}=1, x_{i j}{ }^{l k} \in\{0,1\} \\
\sum_{i \in \mathrm{L}} \sum_{j \in \mathrm{N}} x_{i j}{ }^{l k}=1, x_{i j}^{l k} \in\{0,1\} \\
\sum_{i \in O \cup L} x_{i h}{ }^{k}-\sum_{j \in O \cup L} x_{h j}{ }^{k}=0, h \in O \cup L \\
p_{i}-q \sum_{l \in \mathrm{L}} \sum_{\mathrm{k} \in K} \sum_{j \in \mathrm{O}} c_{i j} x_{i j}{ }^{l k}>0, i \in M \\
\sum_{l \in \mathrm{L}} \sum_{\mathrm{k} \in K} \sum_{\mathrm{i} \in \mathrm{M}} d_{i}^{l k}=\sum_{l \in \mathrm{L}} \sum_{\mathrm{k} \in K} \sum_{j \in N} d_{j}^{l k}
\end{gathered}
$$

In the formula: $\mathrm{M}$ are nodes of a set of the supply, $\mathrm{N}$ are nodes of a set demand, $\mathrm{O}=\mathrm{M} \cup \mathrm{N}$; $\mathrm{L}$ is a collection of parking lots; $\mathrm{K}$ is the set of available transport vehicles; pi is the value of transport; $\mathrm{q}$ is units mileage costs; cij is the distance between $\mathrm{i}$ to $\mathrm{j}$; xijlk is the decision variables, if the vehicle $\mathrm{k}$ starting from yard 1 and drive from $\mathrm{i}$ towards $\mathrm{j}$, take 1 , then take 0 otherwise; di is the traffic of node $\mathrm{i}$.

In the Model: the formula (1) is the revenue - cost function of synergies transportation; the formula (2)means each supply point pickup and only once; the formula (3) refers to each point needs to be and only be shipping once; the formula (4) represents in each node the inflow and outflow of service vehicle is counterpoise; formula(5) represents the value of each supply point transport is more than transportation costs; formula (6) represents the total amount of transport supply and demand set point set point of balance .

For solving vehicle routing problem model, there are a variety of methods, including traditional algorithm and heuristic algorithm. In view of the separation of supply and demand of collaborative distribution problem, paper designs two-stage algorithm to solve. The purpose to design the algorithm is, from local optimal approximate global optimal, and obtain a satisfactory solution. 


\section{Algorithm design}

Generally speaking, the vehicle routing problem can be divided into point path problem and arc routing problem. This paper combine the concept of point and arc , introduces the concept of node - arc discharge.

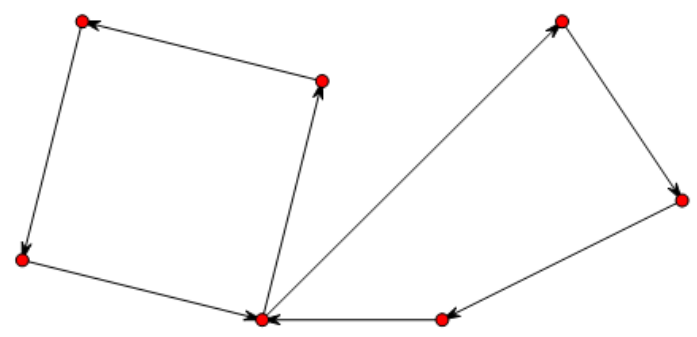

Figure 4. Node routing problem.

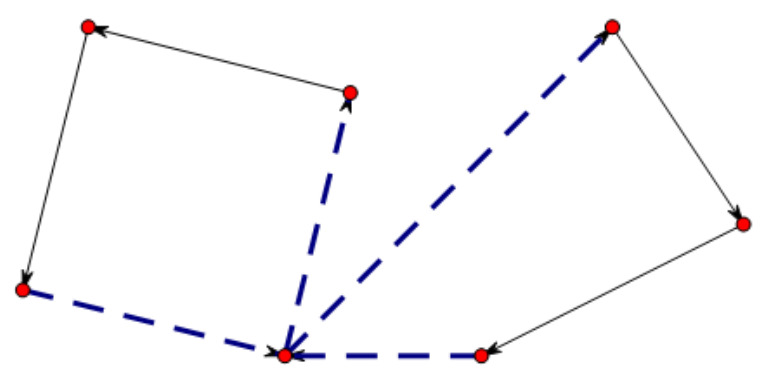

Figure 5. Arc routing problem.

Milk run mode of collaborative transportation problem is belong to the yard vehicle scheduling problem, the article considers the perspective of transportation benefits, establishes the income - cost model, and put forward the thinking of solving the two-phase algorithm on the basis of this. The first stage it introduces the concept of node - arc discharge and split all supply center to each depot, then set the stage and the state, using the dynamic programming algorithm for optimal route of pick up; The second phase uses geometric method by covering arc will delivery routes connect circuits.

The rationality of two phase algorithm is that the supply notes are always far away from the demand notes, the finally pick up location of vehicle changes have less effect on the delivery path length, just as the train passed through a little way, and the distance of the train between the reference has not changed much, this can be explained from the Angle of the differential.

\subsection{The first phase of dynamic programming}

First of all, illustrates the concept of node - arc discharge, This concept is a comprehensive supplier of transport and yard to transport mileage, and the supplier. To transfer the traffic of the node and arc mileage united into a quantity of transportation, need a unified dimensional. The supplier of goods on the basis of size, weight, turning traffic into transport value, The cost of transportation is equal to the yard to supply level between arc length multiplied by the unit transportation cost, Transport value and the difference between the cost of transportation, is the number of arc of the node - arc discharge.

The next major is to determine a collection of major yard service node. A yard $11,12, \ldots \ldots, 1$, Supply nodes $\mathrm{m} 1, \mathrm{~m} 2, \ldots \ldots, \mathrm{mj}$, Supply node behavior, as a yard, the node - arc flow matrix of the:

$$
\left[\begin{array}{llll}
a_{11} & a_{12} & \cdots \cdots & a_{1 j} \\
a_{21} & a_{22} & \cdots \cdots & a_{2 j} \\
\cdots \cdots \cdots \cdots \cdots \cdots & \cdots \cdots \cdots \\
a_{i 1} a_{i 2} & \cdots \cdots & a_{i j}
\end{array}\right]
$$

Determine the set of rules is as follows: For each line in the matrix of the node - arc discharge, take one of the biggest, and divided into corresponding yard, it can be divided all supply nodes in turn.

To determine the significance of the collection is that each station is responsible for the pickup service outlets are transportation value larger nodes, which will be a big problem is decomposed into several small problems.

Do not break general, a yard 11 service node set $\{\mathrm{m} 1, \ldots \ldots, \mathrm{mk}\}$, if expressed in position state, this problem is a typical multistage decision problem, its initial state is 11 , according to the model shows that the $\mathrm{k}$ status to the arrangement of $\mathrm{m} 1 \sim \mathrm{mk}$, which is similar to the calculation of the determinant rule.

\subsection{The second stage geometry method}

After the completion of the first stage of the unstuffing need to arrange delivery of each demand point. A yard li finally pickup node for mj is Michael Jackson, mj is the starting point of delivery, delivery after the return to li, pickup route and route of delivery constitute a loop.

According to the geometric method principle, circuit obtuse Angle in the polygon, the more the greater the Angle, then this polygon Zhou Chang small, easy to understand its rationality from the Angle of mathematics. Setting up a yard is responsible for the delivery of the demand points for $\mathrm{n}$, combining with the cosine theorem, improved geometric method steps are given:

(1) For each station, responsible for pickup outlets, and responsible for the delivery of the demand points, lists its geographical location, for convenience, expressed in plane rectangular coordinates;

(2) Based on cosine function is monotone decreasing in the $0 \sim \pi$ features, To calculate the cosine of every node in (1) based on any two nodes, and the smallest to the $A n+2 n$ cosine value, more nodes in the circumstances, there can be realized by programming;

(3) According to cosine list select nodes and the corresponding edges, since the rules are as follows: select a node and two nodes, in the list of cosine cross to the rest of the cosine value associated with this node, then choose not to scratch the smallest cosine values and the corresponding node, until form a loop.

\section{Analysis of example}

The process of algorithm design fully considers the background of the problem and the applicable scope, and targeted to the filtered solution, make it meet the requirements of the model. Test of practical examples for the rationality of the model and algorithm is indispensable. 
To analyze the feasibility and validity of the model, and constructed two yard, supply level and requirements on the separation of small example, yard location L1(8, $10)$, L2 $(16,16)$, unit mileage charge set to 1 , the fewer number of yard, assuming the same goods to different transport value equal to the yard, each supply level is set to 50 .

Table 1. Supplier point information

\begin{tabular}{|l|l|l|l|l|l|l|l|}
\hline A & B & C & D & E & F & G & H \\
\hline 4,4 & 8,5 & 6,12 & 18,12 & 12,12 & 16,10 & 12,18 & 18,20 \\
\hline
\end{tabular}

Table 2. Demand point information

\begin{tabular}{|l|l|l|l|l|l|l|l|}
\hline A & B & C & D & E & F & G & H \\
\hline 4,4 & 8,5 & 6,12 & 18,12 & 12,12 & 16,10 & 12,18 & 18,20 \\
\hline
\end{tabular}

So $\mathrm{L} 1$ is responsible for the collection of pickup outlets for $\{\mathrm{A}, \mathrm{B}, \mathrm{C}, \mathrm{E}\}, \mathrm{L} 2$ is responsible for the collection of pickup outlets for $\{\mathrm{D}, \mathrm{F}, \mathrm{G}, \mathrm{H}\}$. This is oneway traveling salesman problem; By traveling salesman problem of dynamic programming method, we know that smaller case can use the discrete problem in dynamic programming method for the optimal solution. It is easily obtained that in the supplier concentration area, the first optimal path for $\mathrm{L} 1 \rightarrow \mathrm{B} \rightarrow \mathrm{A} \rightarrow \mathrm{C} \rightarrow \mathrm{E}$, the second optimal path for the $\mathrm{L} 2 \rightarrow \mathrm{G} \rightarrow \mathrm{H} \rightarrow \mathrm{D} \rightarrow \mathrm{F}$.

Next is the route of delivery. Respectively by the end of each unstuffy as a starting point, to deliver goods is regard as the starting point for unstuffy as the end of the delivery operation so as to forming a loop.

Improvement principle of geometric method is established on the basis of Vogel method and geometric method. The establishment of the cosine table make delivery of the nodes selected in turn becomes simple.

Every time when choose a node, we can make the next step of alternative nodes greatly reduced. Delivery can be obtained by applying the method of geometric path, and take the order form a loop.

Loop1 : $\mathrm{L} 1 \rightarrow \mathrm{B} \rightarrow \mathrm{A} \rightarrow \mathrm{C} \rightarrow \mathrm{E} \rightarrow \mathrm{e} \rightarrow \mathrm{c} \rightarrow \mathrm{b} \rightarrow \mathrm{a} \rightarrow \mathrm{L} 1$, The total mileage is 92.94 ;

Loop2: $\mathrm{L} 2 \rightarrow \mathrm{G} \rightarrow \mathrm{H} \rightarrow \mathrm{D} \rightarrow \mathrm{F} \rightarrow \mathrm{f} \rightarrow \mathrm{g} \rightarrow \mathrm{h} \rightarrow \mathrm{d} \rightarrow \mathrm{L} 2, \quad$ The total mileage is 83.26 . So The cooperative transport income is 223.8 .

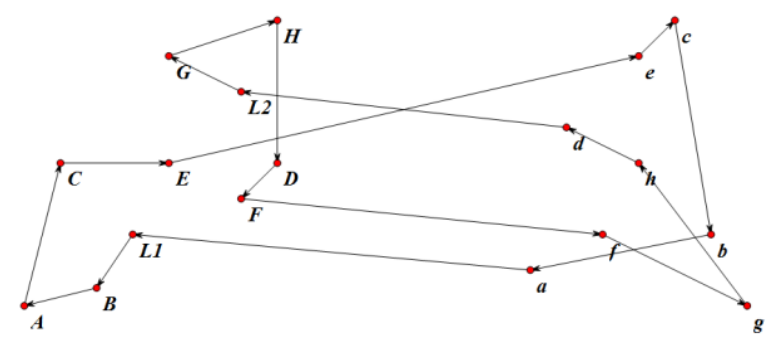

Figure 6. Coordinated transport vehicle trajectory.

\section{Conclusion}

The object of this study is supply level and demand point separation gathered collaborative transportation problem, considering the subject need to provide transportation service to ensure profitability, Therefore, from the perspective of income - cost model is established. According to the complexity of multiple yard to introduce the concept of node - arc discharge and supply level corresponding to yard can be divided into several collections, the essence of this idea is a NP problem into several small problems under certain conditions, to approach the global optimal solution by local optimum.

The design of the two-phase algorithm improves the dynamic programming method and geometry method. Further research can be from the time window, different models such as the angle of the multi-objective optimization.

Collaborative transportation is a new mode of transportation, which is a very complicated system; there are many factors related to it, the problems that need to be solved are very complex and difficult. With the unceasing change of environment, it will also appear new features and problems in this field. We can also coordinate transport problems in the research of the subsequent study combined with other problems. Such as collaborative transportation problem and the profit distribution problems in combination with in-depth study, or combination coordinated transport and cooperative inventory problem, research collaborative logistics oriented collaborative management of inventory and transportation problem is also a very meaningful study direction.

\section{References}

1. R. Liu, Z. Jiang, F. Chen, Journal of Shanghai jiaotong University 3, 43, 455-459, (2009)

2. $\mathrm{K}$. Wen, $\mathrm{Z}$. $\mathrm{Xu}$, Industrial Engineering and Management 12, 19, 51-56, (2014)

3. K. Wen, K. Zhu, G. Liu, Journal of Systems \& Management 1, 24, 38-42, (2015)

4. S. A. Alumur, B. Y. Kara, O. E. Karasan, Omega 40, 6, 927-939, (2012)

5. J. Puerto, A. B. Ramos, Computers \& Operations Research 38, 2, 559-570, (2011)

6. K. Wen, K. Zhu, G. Liu, Chinese Journal of Management Science 1, 23, 135-140, (2015)

7. M. Liu, L. Zhao, Control and Decision 1, 26, 96-100, (2011)

8. W. Tan, Q. Wen, Mathematics in Practice and Theory 12, 45, 235-242, (2015) 